\title{
Reflected backward doubly stochastic differential equations with discontinuous generator
}

\author{
Auguste Aman*and Jean Marc Owo ${ }^{\dagger}$ \\ U.F.R Mathématiques et informatique, Université de Cocody, \\ 582 Abidjan 22, Côte d'Ivoire
}

\begin{abstract}
In this note, we study one-dimensional reflected backward doubly stochastic differential equations (RBDSDEs) with one continuous barrier and discontinuous generator (left-or right-continuous). By a comparison theorem establish here for RBDSDEs, we provide a minimal or a maximal solution to RBDSDEs.
\end{abstract}

AMS Subject Classification: $60 \mathrm{H} 15 ; 60 \mathrm{H} 20$

Keywords: Reflected backward doubly stochastic differential equation, discontinuous generator, comparison theorem.

\section{Introduction}

Backward doubly stochastic differential equations (BDSDEs for short) are equations with two different directions of stochastic integrals, i.e., the equations involve both a standard (forward) stochastic integral $d W_{t}$ and a backward stochastic integral $\overleftarrow{d B}_{t}:$ for $t \in[0, T]$

$$
Y_{t}=\xi+\int_{t}^{T} f\left(s, Y_{s}, Z_{s}\right) d s+\int_{t}^{T} g\left(s, Y_{s}, Z_{s}\right) \overleftarrow{d B}_{s}-\int_{t}^{T} Z_{s} d W_{s}
$$

where $\xi$ is a random variable termed the terminal condition, $f: \Omega \times[0, T] \times \mathbb{R}^{k} \times \mathbb{R}^{d} \rightarrow \mathbb{R}$, $g: \Omega \times[0, T] \times \mathbb{R}^{k} \times \mathbb{R}^{d} \rightarrow \mathbb{R}^{l}$ are two jointly measurable processes, $W$ and $B$ are two mutually independent standard Brownian motion, with values, respectively in $\mathbb{R}^{d}$ and $\mathbb{R}^{l}$. This kind of equations has been introduced by Pardoux and Peng [4] in 1994. A solution of that equation is a couple of jointly measurable processes $(Y, Z)$ with values in $\mathbb{R}^{k} \times \mathbb{R}^{d}$ which mainly satisfies Eq. (1.1). The authors have proved an existence and unique solution when $f$ and $g$ are uniform Lipschitz. They also showed that BDSDEs can produce a probabilistic representation for solutions to some quasi-linear stochastic partial differential equations. Unfortunately, the uniform Lipschitz condition cannot be satisfied in many applications. Many authors have attempted to relax this condition on the coefficients $f$ and $g$.

\footnotetext{
*augusteaman5@yahoo.fr, corresponding author

†owo_jm@yahoo.fr
} 
For instance, Shi et al. [6] weakened the uniform Lipschitz assumptions to linear growth and continuous conditions by virtue of the comparison theorem introduced by themselves. They obtain the existence of solutions to Eq. (1.1) but without uniqueness. Recently, N'zi and Owo [3] have proved an existence solution to Eq. 11.1] when $f$ is discontinuous in $y$ and continuous in $z$.

In this note, we study the now well-know reflected backward doubly stochastic differential equations (RBDSDEs for short):

$$
Y_{t}=\xi+\int_{t}^{T} f\left(s, Y_{s}, Z_{s}\right) d s+\int_{t}^{T} g\left(s, Y_{s}, Z_{s}\right) \overleftarrow{d B}_{s}+K_{T}-K_{t}-\int_{t}^{T} Z_{s} d W_{s}, 0 \leq t \leq T
$$

We establish a comparison theorem for this kind of BDSDEs which help us to derive a maximal and a minimal solution when the generator $f$ is discontinuous. Our work is based to a recent paper of Bahlali et al. [1]. They have proved that Eq. (1.2) has almost one solution and also a maximal and a minimal solution when the generator $f$ is continuous in $y$ and $z$.

The paper is organized as follows. In section 2, we give some notations, definitions and assumptions. Section 3 deals with our main results.

\section{Notations, definitions and assumptions}

Let $(\Omega, \mathcal{F}, \mathbb{P})$ be a probability space in which are defined all the processes considered in the sequel. The Euclidean norm of a vector $x \in \mathbb{R}^{k},(k \geq 2)$ will be denoted by $\|x\|$.

For each $t \in[0, T]$, we define $\mathcal{F}_{t} \triangleq \mathcal{F}_{t}^{W} \vee \mathcal{F}_{t, T}^{B}$, where for any process $\left\{\eta_{t} ; t \in[0, T]\right\}$ and any $0 \leq s \leq t \leq T ; \mathcal{F}_{s, t}^{\eta}=\sigma\left\{\eta_{r}-\eta_{s} ; s \leq r \leq t\right\} \vee \mathcal{N}, \mathcal{F}_{t}^{\eta}=\mathcal{F}_{0, t}^{\eta} ; \mathcal{N}$ denote the class of $\mathbb{P}$-null sets of $\mathcal{F}$.

Note that $\left\{\mathcal{F}_{0, t}^{W}, t \in[0, T]\right\}$ is an increasing filtration and $\left\{\mathcal{F}_{t, T}^{B}, t \in[0, T]\right\}$ is a decreasing filtration, and the collection $\left\{\mathcal{F}_{t}, t \in[0, T]\right\}$ is neither increasing nor decreasing so it does not constitute a filtration.

For any $n \in \mathbb{N}$, let $\mathcal{M}^{2}\left(0, T, \mathbb{R}^{n}\right)$ denote the set of ( class of $d \mathbb{P} \otimes d t$ a.e. equal) $n$ dimensional jointly measurable random processes $\left\{\varphi_{t} ; 0 \leq t \leq T\right\}$ which satisfy:

(i) $\|\varphi\|_{\mathscr{M}^{2}}^{2}=\mathbb{E}\left(\int_{0}^{T}\left|\varphi_{t}\right|^{2} d t\right)<\infty$

(ii) $\varphi_{t}$ is $\mathcal{F}_{t}$-measurable, for a.e. $t \in[0, T]$.

We denote by $\mathcal{S}^{2}\left([0, T], \mathbb{R}^{n}\right)$ the set of continuous $n$-dimensional random processes which satisfy:

(i) $\|\varphi\|_{\mathcal{S}^{2}}^{2}=\mathbb{E}\left(\sup _{0 \leq t \leq T}\left|\varphi_{t}\right|^{2}\right)<\infty$

(ii) $\varphi_{t}$ is $\mathcal{F}_{t}$-measurable, for any $t \in[0, T]$.

Definition 2.1. A solution of a RBDSDE is a triple of processes $(Y, Z, K)$ which satisfies Eq. (1.2) and such that: 
(i) $(Y, Z, K) \in \mathcal{S}^{2}([0, T], \mathbb{R}) \times \mathcal{M}^{2}\left(0, T, \mathbb{R}^{d}\right) \times \mathrm{L}^{2}\left(\Omega, \mathbb{P}, \mathbb{R}_{+}\right)$,

(ii) $Y_{t} \geq S_{t}$, for any $t \in[0, T]$,

(iii) $K_{t}$ is continuous and increasing process with $K_{0}=0$ and $\int_{0}^{T}\left(Y_{t}-S_{t}\right) d K_{t}=0$.

Definition 2.2. A triple of processes $\left(Y_{*}, Z_{*}, K_{*}\right)\left(\operatorname{resp} .\left(Y^{*}, Z^{*}, K^{*}\right)\right)$ of $\mathcal{S}^{2} \times \mathcal{M}^{2} \times \mathrm{L}^{2}(\Omega)$ is said to be a minimal (resp. a maximal) solution of RBDSDE (1.2) if for any other solution $(Y, Z, K)$ of (1.2), we have $Y_{*} \leq Y$ (resp. $\left.Y \leq Y^{*}\right)$.

In this note, we assume that $f$ satisfies some of the following conditions:

(H0) $f: \Omega \times[0, T] \times \mathbb{R} \times \mathbb{R}^{d} \rightarrow \mathbb{R}$ is jointly measurable satisfies $f(., 0,0) \in \mathcal{M}^{2}(0, T, \mathbb{R})$ and there exists a constant $C>0$ such that for all $\left(t, y_{i}, z_{i}\right) \in[0, T] \times \mathbb{R} \times \mathbb{R}^{d}, i=1,2$ $\left|f\left(t, y_{1}, z_{1}\right)-f\left(t, y_{1}, z_{2}\right)\right| \leq C\left(\left|y_{1}-y_{2}\right|+\left\|z_{1}-z_{2}\right\|\right)$.

(H1) For every $(t, \omega) \in[0, T] \times \Omega$, the map $(y, z) \mapsto f(t, y, z)$ is continuous.

(H2) There exists a process $\varphi . \in \mathcal{M}^{2}(0, T, \mathbb{R})$ with positive values and a positive constant $\kappa>0$ such that $|f(t, y, z)| \leq \varphi_{t}+\kappa(|y|+\|z\|)$, for all $(t, y, z) \in[0, T] \times \mathbb{R} \times \mathbb{R}^{d}$.

(H3) For every $(t, \omega) \in[0, T] \times \Omega, z \in \mathbb{R}^{d}$, the map $y \mapsto f(t, y, z)$ is left-continuous and non-decreasing and for $y \in \mathbb{R}, z \mapsto f(t, y, z)$ is continuous.

(H4) There exists a continuous function $h: \mathbb{R} \times \mathbb{R}^{d} \rightarrow \mathbb{R}$, which satisfies

$|h(y, z)| \leq \kappa(|y|+\|z\|)$ for any $(y, z) \in \mathbb{R} \times \mathbb{R}^{d}$, such that for all $y_{1} \geq y_{2}, t \in[0, T]$, $z_{1}, z_{2} \in \mathbb{R}^{d}$, we have $f\left(t, y_{1}, z_{1}\right)-f\left(t, y_{2}, z_{2}\right) \geq h\left(y_{1}-y_{2}, z_{1}-z_{2}\right)$

Moreover, we assume that:

(H5) The terminal condition $\xi$ belongs to $\mathrm{L}^{2}\left(\Omega, \mathcal{F}_{T}, \mathbb{P}, \mathbb{R}\right)$.

(H6) The obstacle $S$ belongs to $\in \mathcal{S}^{2}([0, T], \mathbb{R})$ such that $S_{T} \leq \xi$ a.s.

(H7) $g: \Omega \times[0, T] \times \mathbb{R} \times \mathbb{R}^{d} \rightarrow \mathbb{R}^{l}$ is jointly measurable satisfies $g(., 0,0) \equiv 0$ and there exist two constants $C>0$ and $0<\alpha<1$ such that for all $t \in[0, T],\left(y_{1}, z_{1}\right),\left(y_{2}, z_{2}\right) \in$ $\mathbb{R} \times \mathbb{R}^{d}$, $\left\|g\left(t, y_{1}, z_{1}\right)-g\left(t, y_{2}, z_{2}\right)\right\|^{2} \leq C\left|y_{1}-y_{2}\right|^{2}+\alpha\left\|z_{1}-z_{2}\right\|^{2}$.

\section{Main results}

Our purpose is to establish an existence of minimal or maximal solution to RBDSDEs (1.2) when parameters $(f, g, \xi, S)$ satisfy $(\mathrm{H} 2)-(\mathrm{H} 7)$.

To attain our goal, we need to establish first the following theorem which is an extension of the existence result established in Bahlali et al. [1].

Theorem 3.1. Assume that (H1)-(H2) and (H5)-(H7) hold. Then, the RBDSDE (1.2) has a solution. Moreover, there is a minimal and a maximal solution to RBDSDE (1.2). 
Proof. We define $f_{n}(t, y, z)=\inf _{u \in \mathbb{Q}}\{f(t, u, z)+n|y-u|\}$, for $n \geq \kappa$. For every $n \geq \kappa, f_{n}$ is uniformly $n$-Lipschitz and $\left(f_{n}\right)$ converges suitably to $f$. Now, as $|f(t, y, z)| \leq \varphi_{t}+\kappa(|y|+$ $\|z\|)$, the rest of is the adaptation of Theorem 3.3 in Bahlali et al. [1] where $|f(t, y, z)| \leq$ $\kappa(1+|y|+\|z\|)$. Therefore it is ommitted.

We also need the following comparison results.

Theorem 3.2 (Comparison with at least one Lipschitz function). Let $g, S^{i}$ and $\xi^{i}(i=1,2)$ satisfy (H5)-(H7). Assume that RBDSDEs $\left(f^{1}, g, \xi^{1}, S^{1}\right)$ and $\left(f^{2}, g, \xi^{2}, S^{2}\right)$ have solutions $\left(Y^{1}, Z^{1}, K^{1}\right)$ and $\left(Y^{2}, Z^{2}, K^{2}\right)$, respectively. Assume moreover that:

(i) $\xi^{1} \leq \xi^{2}$ a.s.,

(ii) $S_{t}^{1} \leq S_{t}^{2}$ a.s., for all $t \in[0, T]$

(iii) $f^{1}$ satisfies (HO) such that $f^{1}\left(t, Y^{2}, Z^{2}\right) \leq f^{2}\left(t, Y^{2}, Z^{2}\right)$ a.s.

(resp. $f^{2}$ satisfies (HO) such that $f^{1}\left(t, Y^{1}, Z^{1}\right) \leq f^{2}\left(t, Y^{1}, Z^{1}\right)$ a.s.).

Then, $Y_{t}^{1} \leq Y_{t}^{2}$ a.s., for all $t \in[0, T]$.

Proof. Applying Itô's formula to $\left|\left(Y_{t}^{1}-Y_{t}^{2}\right)^{+}\right|^{2}$, we have

$$
\begin{aligned}
& \mathbb{E}\left|\left(Y_{t}^{1}-Y_{t}^{2}\right)^{+}\right|^{2}+\mathbb{E} \int_{t}^{T} \mathbf{1}_{\left\{Y_{s}^{1}>Y_{s}^{2}\right\}}\left\|Z_{s}^{1}-Z_{s}^{2}\right\|^{2} d s \\
= & \mathbb{E}\left|\left(\xi^{1}-\xi^{2}\right)^{+}\right|^{2}+2 \mathbb{E} \int_{t}^{T}\left(Y_{s}^{1}-Y_{s}^{2}\right)^{+}\left(f^{1}\left(s, Y_{s}^{1}, Z_{s}^{1}\right)-f^{2}\left(s, Y_{s}^{2}, Z_{s}^{2}\right)\right) d s \\
& +2 \mathbb{E} \int_{t}^{T}\left(Y_{s}^{1}-Y_{s}^{2}\right)^{+}\left(d K_{s}^{1}-d K_{s}^{2}\right)+\mathbb{E} \int_{t}^{T} \mathbf{1}_{\left\{Y_{s}^{1}>Y_{s}^{2}\right\}}\left\|g\left(s, Y_{s}^{1}, Z_{s}^{1}\right)-g\left(s, Y_{s}^{2}, Z_{s}^{2}\right)\right\|^{2} d s .
\end{aligned}
$$

From (i), $\mathbb{E}\left|\left(\xi^{1}-\xi^{2}\right)^{+}\right|^{2}=0$ and from (iii), we have

$$
f^{1}\left(s, Y_{s}^{1}, Z_{s}^{1}\right)-f^{2}\left(s, Y_{s}^{2}, Z_{s}^{2}\right) \leq f^{1}\left(s, Y_{s}^{1}, Z_{s}^{1}\right)-f^{1}\left(s, Y_{s}^{2}, Z_{s}^{2}\right) .
$$

Therefore, from Young inequality, and the fact that $f^{1}$ satisfies $(H 0)$ and $g$ verify $(H 7)$, we get

$$
\begin{aligned}
& \mathbb{E}\left|\left(Y_{t}^{1}-Y_{t}^{2}\right)^{+}\right|^{2}+\mathbb{E} \int_{t}^{T} \mathbf{1}_{\left\{Y_{s}^{1}>Y_{s}^{2}\right\}}\left\|Z_{s}^{1}-Z_{s}^{2}\right\|^{2} d s \\
\leq & \left(\frac{1}{\beta}+\beta C+C\right) \mathbb{E} \int_{t}^{T}\left|\left(Y_{s}^{1}-Y_{s}^{2}\right)^{+}\right|^{2} d s+(\beta C+\alpha) \mathbb{E} \int_{t}^{T} \mathbf{1}_{\left\{Y_{s}^{1}>Y_{s}^{2}\right\}}\left\|Z_{s}^{1}-Z_{s}^{2}\right\|^{2} d s \\
& +2 \mathbb{E} \int_{t}^{T}\left(Y_{s}^{1}-Y_{s}^{2}\right)^{+}\left(d K_{s}^{1}-d K_{s}^{2}\right) .
\end{aligned}
$$

Since $Y_{t}^{1}>S_{t}^{2} \geq S_{t}^{1}$ on the set $\left\{Y_{s}^{1}>Y_{s}^{2}\right\}$ we derive that

$$
\mathbb{E} \int_{t}^{T}\left(Y_{s}^{1}-Y_{s}^{2}\right)^{+}\left(d K_{s}^{1}-d K_{s}^{2}\right)=-\mathbb{E} \int_{t}^{T}\left(Y_{s}^{1}-Y_{s}^{2}\right)^{+} d K_{s}^{2} \leq 0 .
$$


Hence,

$$
\begin{aligned}
& \mathbb{E}\left|\left(Y_{t}^{1}-Y_{t}^{2}\right)^{+}\right|^{2}+\mathbb{E} \int_{t}^{T} \mathbf{1}_{\left\{Y_{s}^{1}>Y_{s}^{2}\right\}}\left\|Z_{s}^{1}-Z_{s}^{2}\right\|^{2} d s \\
\leq & \left(\frac{1}{\beta}+\beta C+C\right) \mathbb{E} \int_{t}^{T}\left|\left(Y_{s}^{1}-Y_{s}^{2}\right)^{+}\right|^{2} d s+(\beta C+\alpha) \mathbb{E} \int_{t}^{T} \mathbf{1}_{\left\{Y_{s}^{1}>Y_{s}^{2}\right\}}\left\|Z_{s}^{1}-Z_{s}^{2}\right\|^{2} d s .
\end{aligned}
$$

Consequently, choosing $0<\beta<\frac{1-\alpha}{C}$ and using Gronwall inequality, we obtain $\mathbb{E} \mid\left(Y_{t}^{1}-\right.$ $\left.Y_{t}^{2}\right)\left.^{+}\right|^{2} \leq 0$. Thus $\left(Y_{t}^{1}-Y_{t}^{2}\right)^{+}=0$ a.s. i.e. $Y_{t}^{1} \leq Y_{t}^{2}$ a.s., $\forall t \in[0, T]$.

Theorem 3.3 (Comparison with at least one continuous function). Let $g, S^{i}$ and $\xi^{i}(i=1,2)$ satisfy (H5)-(H7). Assume that RBDSDEs $\left(f^{1}, g, \xi^{1}, S^{1}\right)$ and $\left(f^{2}, g, \xi^{2}, S^{2}\right)$ have solutions $\left(Y^{1}, Z^{1}, K^{1}\right)$ and $\left(Y^{2}, Z^{2}, K^{2}\right)$, respectively. Assume moreover that:

(i) $\xi^{1} \leq \xi^{2}$ a.s.,

(ii) $S_{t}^{1} \leq S_{t}^{2}$ a.s., for all $t \in[0, T]$,

(iii) $f^{1}$ satisfies (H1)-(H2) such that $f^{1}\left(t, Y^{2}, Z^{2}\right) \leq f^{2}\left(t, Y^{2}, Z^{2}\right)$ a.s. and $\left(Y^{1}, Z^{1}, K^{1}\right)$ is the minimal solution (resp. $f^{2}$ satisfies $(H 1)-(H 2)$ such that $f^{1}\left(t, Y^{1}, Z^{1}\right) \leq f^{2}\left(t, Y^{1}, Z^{1}\right)$ a.s. and $\left(Y^{2}, Z^{2}, K^{2}\right)$ is the maximal solution).

Then, $Y_{t}^{1} \leq Y_{t}^{2}$ a.s., for all $t \in[0, T]$.

Proof. For any fixed $\kappa>0$, let us define

$$
f_{n}^{1}(t, y, z)=\inf _{u \in \mathbb{Q}}\left\{f^{1}(t, u, z)+n|y-u|\right\}, \forall n \geq \kappa .
$$

Hence, for every $n \geq \kappa, f_{n}^{1}$ is uniformly $n$-Lipschitz, linear growth and converges suitably to $f^{1}$ (cf. Lepeltier and San Martin [2]). Then we get from Theorem 3.1 that for every $n \geq \kappa$, $\operatorname{RBDSDE}\left(f_{n}^{1}, g, \xi^{1}, S^{1}\right)$ has a unique adapted solution $\left(Y^{1, n}, Z^{1, n}, K^{1, n}\right)$ which converges to the minimal solution $\left(Y^{1}, Z^{1}, K^{1}\right)$ to the RBDSDE $\left(f^{1}, g, \xi^{1}, S^{1}\right)$ (cf. proof of Theorem 3.3 in Bahlali et al. [1]). Moreover, for all $n \geq \kappa, f_{n}^{1} \leq f^{1}$. Therefore, from (iii), we have $f_{n}^{1}\left(t, Y^{2}, Z^{2}\right) \leq f^{2}\left(t, Y^{2}, Z^{2}\right)$ a.s. Then, by Theorem 3.2, we get $Y^{1, n} \leq Y^{2}$ a.s., for all $n \geq \kappa$. Hence, we have $Y^{1} \leq Y^{2}$.

On the other hand, if we define

$$
f_{n}^{2}(t, y, z)=\sup _{u \in \mathbb{Q}}\left\{f^{2}(t, u, z)-n|y-u|\right\} \forall n \geq \kappa
$$

it is easy to check that for all $n \geq \kappa, f^{1}\left(t, Y^{1}, Z^{1}\right) \leq f^{2}\left(t, Y^{1}, Z^{1}\right) \leq f_{n}^{2}\left(t, Y^{1}, Z^{1}\right)$ and $f_{n}^{2}$ is uniformly $n$-Lipschitz, linear growth and converges suitably to $f^{2}$. Then, applying again Theorem 3.2, $Y^{1} \leq Y^{2, n}$ a.s., for all $n \geq \kappa$, where $\left(Y^{2, n}, Z^{2, n}, K^{2, n}\right)$ is the unique solution to BDSDEs $\left(f_{n}^{2}, g, \xi^{2}, S^{2}\right)$ which converges to $\left(Y^{2}, Z^{2}, K^{2}\right)$, the maximal solution of BDSDEs $\left(f^{2}, g, T, \xi^{2}\right)$ (cf. proof of Theorem 3.3 in Bahlali et al. [1]). Therefore, we get $Y^{1} \leq Y^{2}$ a.s. 
Lemma 3.4. Let $\phi$ belongs in $\mathcal{M}^{2}(0, T ; \mathbb{R})$ and h appear in assumption $(H 5)$. For a continuous function of finite variation $A$ belongs in $\mathrm{L}^{2}(\Omega, \mathbb{P}, \mathbb{R})$ and verifies $A_{0}=0$, we consider the processes $(\bar{Y}, \bar{Z}) \in \mathcal{S}^{2}([0, T], \mathbb{R}) \times \mathcal{M}^{2}\left(0, T, \mathbb{R}^{d}\right)$ such that:

(i) $\bar{Y}_{t}=\xi+\int_{t}^{T}\left[h\left(\bar{Y}_{s}, \bar{Z}_{s}\right)+\phi_{s}\right] d s+\int_{t}^{T} g\left(s, \bar{Y}_{s}, \bar{Z}_{s}\right) \overleftarrow{d B}_{s}+A_{T}-A_{t}-\int_{t}^{T} \bar{Z}_{s} d W_{s}, \quad t \in[0, T]$

(ii) $\int_{0}^{T} \bar{Y}_{s}^{-} d A_{s} \geq 0$

Then, if $\phi_{t} \geq 0$ and $\xi \geq 0$, we have $\bar{Y}_{t} \geq 0, \mathbb{P}$-a.s. $\forall t \in[0, T]$.

Remark 3.5. Let us note that the assumption (ii) in Lemma 3.4 is not a technic hypothesis but becomes natural since we are in our framework i.e $\int_{0}^{T}\left(Y_{S}-S_{S}\right) d K_{s}=0$, where $(Y, Z, K)$ is a solution of

Proof. Applying Itô's formula to $\left|Y_{t}^{-}\right|^{2}$, we have

$$
\begin{aligned}
\mathbb{E}\left|\bar{Y}_{t}^{-}\right|^{2}+\mathbb{E} \int_{t}^{T} \mathbf{1}_{\left\{\bar{Y}_{s}<0\right\}}\left\|\bar{Z}_{s}\right\|^{2} d s= & \mathbb{E}\left|\xi^{-}\right|^{2}-2 \mathbb{E} \int_{t}^{T} \bar{Y}_{s}^{-}\left(h\left(\bar{Y}_{s}, \bar{Z}_{s}\right)+\phi_{s}\right) d s \\
& -2 \mathbb{E} \int_{t}^{T} \bar{Y}_{s}^{-} d A_{s}+\mathbb{E} \int_{t}^{T} \mathbf{1}_{\left\{\bar{Y}_{s}<0\right\}}\left\|g\left(s, \bar{Y}_{s}, \bar{Z}_{s}\right)\right\|^{2} d s .
\end{aligned}
$$

Since $\phi_{t} \geq 0$ and $\xi \geq 0$ and using the fact that $-2 \mathbb{E} \int_{t}^{T} \bar{Y}_{s}^{-} d A_{s} \leq 0$, we derive that $\mathbb{E}\left|\bar{Y}_{t}^{-}\right|^{2}+\mathbb{E} \int_{t}^{T} \mathbf{1}_{\left\{\bar{Y}_{s}<0\right\}}\left\|\bar{Z}_{s}\right\|^{2} d s \leq-2 \mathbb{E} \int_{t}^{T} \bar{Y}_{s}^{-} h\left(\bar{Y}_{s}, \bar{Z}_{s}\right) d s+\mathbb{E} \int_{t}^{T} \mathbf{1}_{\left\{\bar{Y}_{s}<0\right\}}\left\|g\left(s, \bar{Y}_{s}, \bar{Z}_{s}\right)\right\|^{2} d s$.

From $(H 7)$, we get $\|g(s, y, z)\|^{2} \leq C|y|^{2}+\alpha\|z\|^{2}$ which together with $(H 4)$ and Young inequality provide

$\mathbb{E}\left|\bar{Y}_{t}^{-}\right|^{2}+\mathbb{E} \int_{t}^{T} \mathbf{1}_{\left\{\bar{Y}_{s}<0\right\}}\left\|\bar{Z}_{s}\right\|^{2} d s \leq\left(\frac{1}{\beta}+2 \beta \kappa^{2}+C\right) \mathbb{E} \int_{t}^{T}\left|\bar{Y}_{s}^{-}\right|^{2} d s+\left(2 \beta \kappa^{2}+\alpha\right) \mathbb{E} \int_{t}^{T} \mathbf{1}_{\left\{\bar{Y}_{s}<0\right\}}\left\|\bar{Z}_{s}\right\|^{2} d s$.

Therefore, choosing $0<\beta<\frac{1-\alpha}{2 \mathrm{~K}^{2}}$ and using Gronwall inequality, we obtain $\bar{Y}_{t}^{-}=0 \mathbb{P}$-a.s. $\forall t \in[0, T]$, which implies that $\bar{Y}_{t} \geq 0 \mathbb{P}$-a.s. $\forall t \in[0, T]$.

Now, we are ready to prove our main result.

Theorem 3.6. Under assumptions (H2)-(H7), the RBDSDE (1.2) has at least one solution. Also, there is a minimal solution $(\underline{y}, \underline{z}, \underline{k})$ to RBDSDE (1.2).

Proof. By virtue of Theorem 3.1, let consider the processes $\left(y^{0}, z^{0}, k^{0}\right),\left(\tilde{y}^{0}, \tilde{z}^{0}, \tilde{k}^{0}\right)$ and the sequence of processes $\left\{\left(y^{n}, z^{n}, k^{n}\right)\right\}_{n \geq 1}$ respectively minimal solution of the following RBDSDE: for all $t \in[0, T]$,

$$
\left\{\begin{array}{l}
y_{t}^{0}=\xi+\int_{t}^{T}\left(-\kappa\left|y_{s}^{0}\right|-\kappa\left\|z_{s}^{0}\right\|-\varphi_{s}\right) d s+k_{T}^{0}-k_{t}^{0}+\int_{t}^{T} g\left(s, y_{s}^{0}, z_{s}^{0}\right) \overleftarrow{d B}_{s}-\int_{t}^{T} z_{s}^{0} d W_{s} \\
y_{t}^{0} \geq S_{t} \\
\int_{0}^{T}\left(y_{s}^{0}-S_{s}\right) d k_{s}^{0}=0
\end{array}\right.
$$




$$
\left\{\begin{array}{l}
\tilde{y}_{t}^{0}=\xi+\int_{t}^{T}\left(\kappa\left|\tilde{y}_{s}^{0}\right|+\kappa|| \tilde{z}_{s}^{0} \|+\varphi_{s}\right) d s+\tilde{k}_{T}^{0}-\tilde{k}_{t}^{0}+\int_{t}^{T} g\left(s, \tilde{y}_{s}^{0}, \tilde{z}_{s}^{0}\right) \overleftarrow{d B}_{s}-\int_{t}^{T} \tilde{z}_{s}^{0} d W_{s}, t \in[0, T] \\
\tilde{y}_{t}^{0} \geq S_{t}, \quad t \in[0, T] \\
\int_{0}^{T}\left(\tilde{y}_{s}^{0}-S_{s}\right) d \tilde{k}_{s}^{0}=0 .
\end{array}\right.
$$

and

$$
\left\{\begin{array}{l}
y_{t}^{n}=\xi+\int_{t}^{T}\left(f\left(s, y_{s}^{n-1}, z_{s}^{n-1}\right)+h\left(y_{s}^{n}-y_{s}^{n-1}, z_{s}^{n}-z_{s}^{n-1}\right)\right) d s+k_{T}^{n}-k_{t}^{n}+\int_{t}^{T} g\left(s, y_{s}^{n}, z_{s}^{n}\right) \overleftarrow{d B_{s}}-\int_{t}^{T} z_{s}^{n} d W_{s} \\
y_{t}^{n} \geq S_{t} \\
\int_{0}^{T}\left(y_{s}^{n}-S_{s}\right) d k_{s}^{n}=0 .
\end{array}\right.
$$

To complete the proof, it's suffice to show that the sequence $\left(y^{n}, z^{n}, k^{n}\right)$ converges to a limit $(\underline{y}, \underline{z}, \underline{k})$ which is the minimal solution of RBDSDE (1.2). In this end, we shall first prove that for any $n \geq 0$,

$$
y_{t}^{n} \leq y_{t}^{n+1} \leq \tilde{y}_{t}^{0}, \quad \mathbb{P} \text {-a.s. } \forall t \in[0, T] .
$$

For $n \geq 0$, we set $\left(y_{t}^{n+1, n}, z_{t}^{n+1, n}, k_{t}^{n+1, n}\right)=\left(y_{t}^{n+1}-y_{t}^{n}, z_{t}^{n+1}-z_{t}^{n}, k_{t}^{n+1}-k_{t}^{n}\right)$, which satisfies the following equation:

$$
\begin{aligned}
y_{t}^{n+1, n}= & \int_{t}^{T}\left(h\left(y_{s}^{n+1, n}, z_{s}^{n+1, n}\right)+\phi_{s}^{n}\right) d s+k_{T}^{n+1, n}-k_{t}^{n+1, n}+\int_{t}^{T} g^{n}\left(s, y_{s}^{n+1, n}, z_{s}^{n+1, n}\right) \overleftarrow{d B}_{s} \\
& -\int_{t}^{T} z_{s}^{n+1, n} d W_{s},
\end{aligned}
$$

where $g^{n}(t, y, z)=g\left(t, y+y_{t}^{n}, z+z_{t}^{n}\right)-g\left(t, y_{t}^{n}, z_{t}^{n}\right), \forall n \geq 0, \phi_{s}^{0}=f\left(s, y_{s}^{0}, z_{s}^{0}\right)+\kappa\left|y_{s}^{0}\right|+\kappa\left\|z_{s}^{0}\right\|+\varphi_{s}$ and $\phi_{s}^{n}=f\left(s, y_{s}^{n}, z_{s}^{n}\right)-f\left(s, y_{s}^{n-1}, z_{s}^{n-1}\right)-h\left(y_{s}^{n}-y_{s}^{n-1}, z_{s}^{n}-z_{s}^{n-1}\right), n \geq 1$. According to it definition, one can show that $\phi^{0}$ and $g^{n}, \forall n \geq 0$ satisfy all assumptions of Lemma3.4. Moreover, since $k_{t}^{n}$ is a continuous and increasing process, for all $n \geq 0, k^{n+1, n}$ is a continuous processes of finite variation and, using the same argument as one appear in [1], on can show that

$$
\int_{0}^{T}\left(y_{t}^{n+1, n}\right)^{-} d k_{t}^{n+1, n}=\int_{0}^{T}\left(y_{t}^{n+1}-y_{t}^{n}\right)^{-} d k_{t}^{n+1} \geq 0 .
$$

Therefore, it follows from Lemma 3.4 that $y_{t}^{1,0} \geq 0$ a.s., i.e. $y_{t}^{0} \leq y_{t}^{1}$, a.s.for all $t \in[0, T]$. Let us suppose that there exists $n \geq 1$ such that $y_{t}^{n-1} \leq y_{t}^{n}$. Then, for such $n, \phi^{n}$ satisfies assumption of Lemma 3.4 from which, we obtain $y_{t}^{n} \leq y_{t}^{n+1}$, a.s., for all $t \in[0, T]$. Finally, for all $n \geq 0, y_{t}^{n} \leq y_{t}^{n+1}$ a.s. for all $t \in[0, T]$.

Setting $\left(\tilde{y}_{t}^{0, n}, \tilde{z}_{t}^{0, n}, \tilde{k}_{t}^{0, n}\right)=\left(\tilde{y}_{t}^{0}-y_{t}^{n}, \tilde{z}_{t}^{0}-z_{t}^{n}, \tilde{k}_{t}^{0}-k_{t}^{n}\right)$, we check similarly as above that for all $n \geq 0, \tilde{y}_{t}^{0, n} \geq 0$ a.s., for all $t \in[0, T]$, i.e. for all $n \geq 0, y_{t}^{n} \leq \tilde{y}_{t}^{0}$, a.s., for all $t \in[0, T]$. Thus, we have for all $n \geq 0$,

$$
y_{t}^{n} \leq y_{t}^{n+1} \leq \tilde{y}_{t}^{0}, \quad \mathbb{P} \text {-a.s. } \forall t \in[0, T] .
$$

Moreover, since $\left|y_{t}^{n}\right| \leq \max \left(\left|\tilde{y}_{t}^{0}\right|,\left|y_{t}^{0}\right|\right), \forall t \in[0, T]$ we have

$$
\sup _{n} \mathbb{E}\left(\sup _{0 \leq t \leq T}\left|y_{t}^{n}\right|^{2}\right) \leq \max \left(\mathbb{E}\left(\sup _{0 \leq t \leq T}\left|\tilde{y}_{t}^{0}\right|^{2}\right), \mathbb{E}\left(\sup _{0 \leq t \leq T}\left|y_{t}^{0}\right|^{2}\right)\right)<+\infty .
$$


Therefore, we deduce from the dominated convergence theorem that $\left(y_{s}^{n}\right)_{n \geq 0}$ converges in $\mathcal{S}^{2}([0, T], \mathbb{R})$ to a limit $y$.

On the other hand, by virtue of Itô's formula, we have

$$
\begin{aligned}
\mathbb{E}\left(\left|y_{0}^{n+1}\right|^{2}+\int_{0}^{T}\left\|z_{s}^{n+1}\right\|^{2} d s\right)= & \mathbb{E}|\xi|^{2}+2 \mathbb{E} \int_{0}^{T} y_{s}^{n+1}\left(f\left(s, y_{s}^{n}, z_{s}^{n}\right)+h\left(y_{s}^{n+1}-y_{s}^{n}, z_{s}^{n+1}-z_{s}^{n}\right)\right) d s \\
& +2 \mathbb{E} \int_{0}^{T} y_{s}^{n+1} d k_{s}^{n+1}+\mathbb{E} \int_{0}^{T}\left\|g\left(s, y_{s}^{n+1}, z_{s}^{n+1}\right)\right\|^{2} d s
\end{aligned}
$$

From (H2), (H4), (H7) and Young inequalities, we get for any $\gamma, \sigma>0$,

$$
\begin{aligned}
y_{s}^{n+1}\left(f\left(s, y_{s}^{n-1}, z_{s}^{n-1}\right)+h\left(y_{s}^{n}-y_{s}^{n-1}, z_{s}^{n}-z_{s}^{n-1}\right)\right) \leq & \left|y_{s}^{n+1}\right| \varphi_{s}+\kappa\left|y_{s}^{n+1}\right|\left(2\left|y_{s}^{n}\right|+2\left\|z_{s}^{n}\right\|+\left|y_{s}^{n+1}\right|+\left\|z_{s}^{n+1}\right\|\right) \\
\leq & \left(\frac{1}{2}+\kappa^{2}+\frac{2 \kappa^{2}}{\gamma}+\kappa+\frac{\kappa^{2}}{2 \sigma}\right)\left|y_{s}^{n+1}\right|^{2}+\left|y_{s}^{n}\right|^{2}+\frac{\gamma}{2}\left\|z_{s}^{n}\right\|^{2} \\
& +\frac{\sigma}{2}\left\|z_{s}^{n+1}\right\|^{2}+\frac{1}{2}\left|\varphi_{s}\right|^{2}, \\
\left\|g\left(s, y_{s}^{n+1}, z_{s}^{n+1}\right)\right\|^{2} \leq & C\left|y_{s}^{n+1}\right|^{2}+\alpha\left\|z_{s}^{n+1}\right\|^{2} .
\end{aligned}
$$

Using again Young inequality, we have for any $\beta>0$,

$$
2 \mathbb{E} \int_{0}^{T} y_{s}^{n+1} d k_{s}^{n+1}=2 \int_{0}^{T} S_{s} d k_{s}^{n+1} \leq \frac{1}{\beta} \mathbb{E}\left(\sup _{0 \leq t \leq T}\left|S_{s}\right|^{2}\right)+\beta \mathbb{E}\left(k_{T}^{n+1}\right)^{2}
$$

Therefore, there exists a constant $C_{1}$ independent of $n$ such that for any $\gamma, \sigma>0$, we derive

$$
\mathbb{E} \int_{0}^{T}\left\|z_{s}^{n+1}\right\|^{2} d s \leq C_{1}+(\sigma+\alpha) \mathbb{E} \int_{0}^{T}\left\|z_{s}^{n+1}\right\|^{2} d s+\gamma \mathbb{E} \int_{0}^{T}\left\|z_{s}^{n}\right\|^{2} d s+\beta \mathbb{E}\left|k_{T}^{n+1}\right|^{2} .
$$

Moreover, since

$$
\begin{aligned}
k_{T}^{n+1}=y_{0}^{n+1}- & \xi-\int_{0}^{T}\left(f\left(s, y_{s}^{n}, z_{s}^{n}\right)+h\left(y_{s}^{n+1}-y_{s}^{n}, z_{s}^{n+1}-z_{s}^{n}\right)\right) d s-\int_{0}^{T} g\left(s, y_{s}^{n+1}, z_{s}^{n+1}\right) \overleftarrow{d B}_{s} \\
& +\int_{0}^{T} z_{s}^{n+1} d W_{s}, t \in[0, T],
\end{aligned}
$$

it follows from Hölder and BDG's inequalities and the properties on $f, h$ and $g$ that there exists $C_{2}$ independent of $n$ such that

$$
\mathbb{E}\left(k_{T}^{n+1}\right)^{2} \leq C_{2}+c \mathbb{E} \int_{0}^{T}\left(\left\|z_{s}^{n}\right\|^{2}+\left\|z_{s}^{n+1}\right\|^{2}\right) d s
$$

According to (3.7) and (3.8) and choosing $\sigma>0$ and $\beta>0$ such that $0<\sigma+\beta c<1-\alpha$, we derive for any $\gamma>0, n \geq 0$

$$
\mathbb{E} \int_{0}^{T}\left\|z_{s}^{n+1}\right\|^{2} d s \leq \frac{\Lambda}{1-\alpha-\sigma-\beta c}+\frac{\gamma+\beta c}{1-\alpha-\sigma-\beta c} \mathbb{E} \int_{0}^{T}\left|z_{s}^{n}\right|^{2} d s,
$$

which provide by iteration

$$
\begin{aligned}
\mathbb{E} \int_{0}^{T}\left\|z_{s}^{n+1}\right\|^{2} d s \leq & \frac{\Lambda}{1-\alpha-\sigma-\beta c} \sum_{i=0}^{n-1}\left(\frac{\gamma+\beta c}{1-\alpha-\sigma-\beta c}\right)^{i} \\
& +\left(\frac{\gamma+\beta c}{1-\alpha-\sigma-\beta c}\right)^{n} \mathbb{E} \int_{0}^{T}\left\|z_{s}^{0}\right\|^{2} d s .
\end{aligned}
$$


Choosing $\gamma>0$ (for example, one can take: $0<\sigma<\frac{1-\alpha}{2}, 0<\beta<\frac{1-\alpha}{4 c}$ and $0<\gamma<$ $1-\alpha-\sigma-2 \beta c)$ such that $0<\frac{\gamma+\beta c}{1-\alpha-\sigma-\beta c}<1$ and noting that $\mathbb{E} \int_{0}^{T}\left\|z_{s}^{0}\right\|^{2} d s<\infty$, we obtain

$$
\sup _{n \geq 0} \mathbb{E} \int_{0}^{T}\left\|z_{s}^{n}\right\|^{2} d s<+\infty
$$

Denoting $\theta_{s}^{n}=f\left(s, y_{s}^{n-1}, z_{s}^{n-1}\right)+h\left(y_{s}^{n}-y_{s}^{n-1}, z_{s}^{n}-z_{s}^{n-1}\right)$, it follows from (3.5) and (3.10) that $\theta_{s}^{n}$ is uniformly bounded in $\mathcal{M}^{2}(0, T, \mathbb{R})$.

Applying again Itô's formula to $\left|y_{t}^{p}-y_{t}^{n}\right|^{2}$, we have

$$
\begin{aligned}
\mathbb{E}\left|y_{t}^{p}-y_{t}^{n}\right|^{2}+\mathbb{E} \int_{t}^{T}\left\|z_{s}^{p}-z_{s}^{n}\right\|^{2} d s= & 2 \mathbb{E} \int_{t}^{T}\left(y_{s}^{p}-y_{s}^{n}\right)\left(\theta_{s}^{p}-\theta_{s}^{n}\right) d s+2 \mathbb{E} \int_{t}^{T}\left(y_{s}^{p}-y_{s}^{n}\right)\left(d k_{s}^{p}-d k_{s}^{n}\right) \\
& +\mathbb{E} \int_{t}^{T}\left\|g\left(s, y_{s}^{p}, z_{s}^{p}-\right) g\left(s, y_{s}^{n}, z_{s}^{n}\right)\right\|^{2} d s .
\end{aligned}
$$

Using the fact that $y_{t}^{n} \geq S_{t}$ for all $t \in[0, T]$ and the identity $\int_{0}^{T}\left(y_{s}^{n}-S_{s}\right) d k_{s}^{n}=0$, we obtain $\mathbb{E} \int_{0}^{T}\left\|z_{s}^{p}-z_{s}^{n}\right\|^{2} d s \leq 2 \mathbb{E} \int_{0}^{T}\left(y_{s}^{p}-y_{s}^{n}\right)\left(\theta_{s}^{p}-\theta_{s}^{n}\right) d s+\mathbb{E} \int_{0}^{T}\left\|g\left(s, y_{s}^{p}, z_{s}^{p}\right)-g\left(s, y_{s}^{n}, z_{s}^{n}\right)\right\|^{2} d s$.

Therefore, by virtue of Hölder's inequality and (H7), we obtain

$$
(1-\alpha) \mathbb{E} \int_{0}^{T}\left\|z_{s}^{p}-z_{s}^{n}\right\|^{2} d s \leq 4\left(\sup _{n \geq 0}\left\|\theta^{n}\right\|_{\mathcal{M}^{2}}\right)\left(\mathbb{E} \int_{0}^{T}\left|y_{s}^{p}-y_{s}^{n}\right|^{2} d s\right)^{\frac{1}{2}}+C \mathbb{E} \int_{0}^{T}\left|y_{s}^{p}-y_{s}^{n}\right|^{2} d s
$$

which yields that $\left(z^{n}\right)_{n \geq 0}$ is a Cauchy sequence in $\mathcal{M}^{2}\left(0, T, \mathbb{R}^{d}\right)$ so that it converges in $\mathcal{M}^{2}\left(0, T, \mathbb{R}^{d}\right)$ to a limit $\underline{z}$. On the other hand, since $\left(y^{n}, z^{n}\right) \rightarrow(\underline{y}, \underline{z})$ in $\mathcal{M}^{2}\left(\mathbb{R}^{d}\right) \times \mathcal{S}(\mathbb{R})$, then there exists $\left(y^{\prime}, z^{\prime}\right) \in M^{2}\left(\mathbb{R}^{d}\right) \times S(\mathbb{R})$ and a subsequence which we still denote $\left(y^{n}, z^{n}\right)$ such that $\forall n,\left|y^{n}\right|<y^{\prime},\left\|z^{n}\right\|<z^{\prime}$ and $\left(y^{n}, z^{n}\right) \rightarrow(\underline{y}, \underline{z}), d t \times d \mathbb{P}$ a.e. Therefore, from the properties of $f, g$ and $h$, we get for almost all $\omega$,

$$
f\left(t, y_{t}^{n-1}, z_{t}^{n-1}\right)+h\left(y_{t}^{n}-y_{t}^{n-1}, z_{t}^{n}-z_{t}^{n-1}\right) \longrightarrow f\left(t, \underline{y}_{t}, \underline{z}_{t}\right),
$$

$\mathbb{P}$-a.s., for all $t \in[0, T]$ as $n \rightarrow \infty$. Then, it follows by the dominated convergence theorem that

$$
\mathbb{E} \int_{t}^{T}\left|f\left(s, y_{s}^{n-1}, z_{s}^{n-1}\right)+h\left(y_{s}^{n}-y_{s}^{n-1}, z_{s}^{n}-z_{s}^{n-1}\right)-f\left(s, \underline{y}_{s}, \underline{z}_{s}\right)\right|^{2} d s \rightarrow 0
$$

as $n \rightarrow \infty$. On the other hand, by Burkhölder-Davis Gundy inequality,

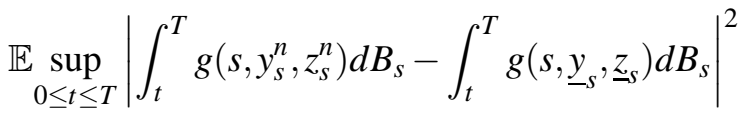

$$
\begin{aligned}
& \leq C \mathbb{E} \int_{0}^{T}\left|y_{s}^{n}-\underline{y}_{s}\right|^{2} d s+\alpha \mathbb{E} \int_{0}^{T}\left\|z_{s}^{n}-\underline{z}_{s}\right\|^{2} d s \underset{n \longrightarrow \infty}{\longrightarrow} 0,
\end{aligned}
$$


and

$$
\underset{0 \leq t \leq T}{\mathbb{E} \sup _{0 \leq t}}\left|\int_{t}^{T} z_{s}^{n} d W_{s}-\int_{t}^{T} \underline{z}_{s} d W_{s}\right|^{2} \leq \mathbb{E} \int_{0}^{T}\left|z_{s}^{n}-\underline{z}_{s}\right|^{2} d s \underset{n \longrightarrow \infty}{\longrightarrow} 0 .
$$

Since, $\left(y^{n}, z^{n}, \theta^{n}\right)$ converges in $\mathcal{S}^{2}([0, T] ; \mathbb{R}) \times \mathcal{M}^{2}\left([0, T] ; \mathbb{R}^{d}\right) \times \mathcal{M}^{2}\left([0, T] ; \mathbb{R}^{d}\right)$ and

$$
\begin{aligned}
\mathbb{E} \sup _{0 \leq t \leq T}\left|k_{t}^{p}-k_{t}^{n}\right|^{2} \leq & \mathbb{E}\left|y_{0}^{p}-y_{0}^{n}\right|^{2}+\mathbb{E} \sup _{0 \leq t \leq T}\left|y_{t}^{p}-y_{t}^{n}\right|^{2}+\mathbb{E} \int_{0}^{T}\left|\theta_{s}^{p}-\theta_{s}^{n}\right|^{2} d s \\
& +\mathbb{E} \sup _{0 \leq t \leq T}\left|\int_{0}^{t}\left(g\left(s, y_{s}^{p}, z_{s}^{p}\right)-g\left(s, y_{s}^{n}, z_{s}^{n}\right)\right) \overleftarrow{d B_{s}}\right|^{2}+\mathbb{E} \sup _{0 \leq t \leq T}\left|\int_{0}^{t}\left(z_{s}^{p}-z_{s}^{n}\right) d W_{s}\right|^{2},
\end{aligned}
$$

for any $n, p \geq 0$, we deduce from Burkhölder-Davis Gundy inequality that

$$
\mathbb{E}\left(\sup _{0 \leq t \leq T}\left|k_{t}^{p}-k_{t}^{n}\right|^{2}\right) \rightarrow 0
$$

as $n, p \rightarrow \infty$. Consequently, there exists a $\mathcal{F}_{t}$-mesurable process $k$ with value in $\mathbb{R}$ such that

$$
\mathbb{E}\left(\sup _{0 \leq t \leq T}\left|k_{t}^{n}-\underline{k}_{t}\right|^{2}\right) \longrightarrow 0
$$

as $n \rightarrow \infty$. Obviously, $\underline{k}_{0}=0$ and $\left\{\underline{k}_{t} ; 0 \leq t \leq T\right\}$ is a non-decreasing and continuous process. From (3.4), we have for all $n \geq 0, y_{t}^{n} \geq S_{t}, \forall t \in[0, T]$, then $\underline{y}_{t} \geq S_{t}, \forall t \in[0, T]$.

On the other hand, from the result of Saisho [5] (1987, p. 465), we have

$$
\int_{0}^{T}\left(y_{s}^{n}-S_{s}\right) d k_{s}^{n} \rightarrow \int_{0}^{T}\left(\underline{y}_{s}-S_{s}\right) d k_{s}
$$

$\mathbb{P}$-a.s. as $n \rightarrow \infty$. Using the identity $\int_{0}^{T}\left(y_{s}^{n}-S_{s}\right) d k_{s}^{n}=0$, for all $n \geq 0$, we obtain

$\int_{0}^{T}\left(\underline{y}_{s}-S_{s}\right) d \underline{k}_{s}=0$. Finally, passing to the limit in $(\underline{3.4)}$, we get that $(\underline{y}, \underline{z}, \underline{k})$ is a solution of the RBDSDE (1.2).

Let $(y, z, k)$ be any solution of the RBDSDE (1.2). By virtue of Theorem 3.3, we have $y^{n} \leq y$, for all $n \geq 0$ and therefore, $\underline{y} \leq y$ i.e., $\underline{y}$ is the minimal solution.

Remark 3.7. We can prove the maximal solution result for BDSDEs (1.2) when the coefficient $f$ is right-continuous and decreasing.

\section{References}

[1] Bahlali, K., Hassani, M., Mansouri, B., and Mrhardy, N. One barrier reflected backward doubly differential equations with continuous generator. C.R. Acad. Sci. Paris, Ser. I 347 (2009), pages 1201-1206.

[2] Lepeltier, J.P and San Martin, J. Backward stochastic differential equations with continuous coefficients. Statist. Probab. Lett, 32 (4): 425-430, 1997. 
[3] N'zi, M. and Owo, J.-M. Backward doubly stochastic differential equations with discontinuous coefficients. Statist. Probab. Lett, 79: 920-926, 2009. doi:10.1016/j.spl.2008.11.011.

[4] Pardoux, E. and Peng, S. Backward doubly stochastic differential equations and systèmes of quasilinear SPDEs. Probab. Theory Related Fields., 98: 209-227, 1994.

[5] Saisho, Y. SDE for multidimensional domains with reflecting boundary. Probab. Theory Related Fields, 74 : 455-477, 1987.

[6] Yufen, S.; Yanling, G. and Kai, L. Comparison theorem of backward doubly stochastic differential equations and application. Stoch. Anal. Appl., 23: no.1, 97-110, 2005. 